\title{
New hemostatic device for grade IV-V liver injury in porcine model: a proof of concept
}

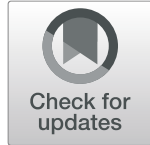

Juan José Segura-Sampedro ${ }^{1,2,3^{*}}$ (D), Cristina Pineño-Flores ${ }^{1,2}$, Andrea Craus-Miguel ${ }^{1,2}$, Rafael Morales-Soriano ${ }^{1,2}$ and Francesc Xavier González-Argente ${ }^{1,2,3}$

\begin{abstract}
Background: The liver is the most injured organ following abdominal trauma. Uncontrolled bleeding remains the main cause of early liver injury-related death, with a mortality rate of $50-54 \%$ in the first $24 \mathrm{~h}$ after admission and with $80 \%$ of operative deaths. Packing and reoperation account for the increased survival in severe liver trauma, and they are recommended for severe liver injuries (grades IV-V).

Perihepatic packing can lead to several potential complications. An excessive packing can cause complications due to abdominal compartment syndrome, while a soft packing may be ineffective, and thus, bleeding can continue inadvertently with the consequent hypovolemic shock and potentially death.

Methods: We designed a new vacuum-based device to perform perihepatic packing without the negative sideeffects of the classic technique. We conducted a prospective pilot feasibility study in a porcine model. We compared the traditional perihepatic packing (PHP) $(n=2)$ with the new VacBagPack device (VBP) $(n=2)$.
\end{abstract}

Results: Both pigs survived with the new device and showed an equivalent outcome to the one that survived in the traditional technique group. Blood tests were similar too. This suggests that VBP could be at least as effective as traditional PHP.

Conclusions: We establish a first step towards the development of a new packing device. A new study with a bigger sample size still in pigs will be conducted. Also, an industrial model of the device is currently in production.

Keywords: Abdominal trauma, Liver injury, Hemostatic device, Damage control

\section{Introduction}

The liver is the most injured organ following abdominal trauma. A major liver injury is the leading cause of death in patients with abdominal trauma, the treatment of which still poses a challenge to surgeons [1-3]. Uncontrolled bleeding remains the main cause of early liver injury-related death, with a mortality rate of $50-54 \%$ in the first $24 \mathrm{~h}$ after admission and with $80 \%$ of operative deaths $[1,4,5]$.

Packing and reoperation account for the increased survival in severe liver trauma in the last decades $[2,6]$.

\footnotetext{
* Correspondence: segusamjj@gmail.com

Part of this work has been presented in the Spanish National Congress of Surgery of 2018.

${ }^{1}$ General \& Digestive Surgery Department, University Hospital Son Espases, Palma de Mallorca, Spain

${ }^{2}$ Health Research Institute of Balearic Islands, Palma de Mallorca, Spain

Full list of author information is available at the end of the article
}

This once revolutionary approach stands now as part of the recommended management for severe liver injuries (grades IV-V) [2, 7].

Although hepatic packing is an extended maneuver, it requires experience to perform it properly and involves several potential complications. An excessive packing can cause complications due to abdominal compartment syndrome [8], while a soft packing may be ineffective and thus bleeding can continue inadvertently with the consequent hypovolemic shock and potentially death $[2,7,9]$.

Even if bleeding control is achieved through this maneuver, subsequent complications such as intra-abdominal abscesses, bleeding after withdrawal, or the loss of compresses within the patient are still common.

The purpose of this work is to test a new device conceived to achieve effective bleeding control after severe liver injury without the previous complications. To do

(c) The Author(s). 2019 Open Access This article is distributed under the terms of the Creative Commons Attribution 4.0 International License (http://creativecommons.org/licenses/by/4.0/), which permits unrestricted use, distribution, and 
so, we compared the classical packing method with the new device in a porcine model.

\section{Material and methods}

We conducted a pilot feasibility study in a porcine model. We compared the traditional perihepatic packing (PHP) $(n=2)$ with the new VacBagPack device (VBP) $(n=2)$.

\section{VacBagPack device design}

This new device consists of a new bag for organ packing that allows compressing the liver in a controlled manner. To that end, the bag is configured to be able to use an external vacuum source to compress the liver via the application of a vacuum, and thus to promote its recovery after a traumatic incident. Furthermore, it may allow determining the volume of blood loss of an organ by connecting a collector to the external vacuum source, which helps determine blood loss at all times [10].

The bag, which has a similar shape to a human liver, may surround the injured portion of the liver. The shape of the bag can be adapted to the wrapped portion of the organ by applying negative pressure, as this allows the bag to compress and thus to adopt the shape of the portion of the organ [10].

The bag is made from a multilayer material that comprises the following elements: an inner layer, an outer layer, and an intermediate layer arranged between the inner and the outer layers [10]. Below, each of these layers is defined in more detail:

a) Inner layer This is a microperforated inner layer to allow applying the vacuum to the liver [10].

b) Outer layer This is a watertight outer layer which surrounds the microperforated inner layer. The watertight outer layer may be flexible and comprise an inlet to apply the vacuum to the intermediate region between the microperforated inner layer and the watertight outer layer. Alternatively, the watertight outer layer may lack a preformed inlet, so that the surgeon may perforate, for example with a scalpel or scissors, the outer layer in the most appropriate region to connect the external vacuum source. In this case, the outer layer may be connected to the external vacuum source using known systems, as a VAC Therapy System from KCI Medical [10].

c) Intermediate layer

This is a porous intermediate layer that fills the intermediate region between the microperforated inner layer and the watertight outer layer. The pores of this layer are precisely interconnected so a fluid can easily be distributed throughout the intermediate layer.
The main objective of this porous intermediate layer is to allow the distribution of the vacuum applied through the outer layer, for example, through its inlet, throughout the entire surface of the inner layer. Because this layer is made from a porous material, it contributes to create a gap between the inner and the outer layers that allows the vacuum to reach all the holes of the inner layer. Furthermore, this configuration also allows the blood extracted from the organ to flow towards the external layer. In addition, the air extracted from the porous intermediate layer along with the effect of the compression of this intermediate layer between the other two layers increase the consistency of the porous layer, which helps hold and compress the liver. Also, the intermediate layer may be made from a compressible and moldable material. The compression of the intermediate layer when a negative pressure is applied uniformly distributes the pressure exerted onto the organ. Moreover, this compressibility also helps control the pressure applied to the organ [10].

When the bag surrounds the liver, this configuration allows the application of vacuum through the watertight outer layer to the intermediate region in order to compress the organ and to extract fluids placed on the surface of the liver. Accordingly, the pressure exerted against the organ may be controlled.

A homemade device was created using compresses, drains, and adhesive plastics as can be seen in the Additional file 1: Video 1.

\section{Animals}

Animal use and procedures were approved by the Research Ethics Committee of the Balearic Islands. The swine model is widely preferred to emulate human trauma $[11,12]$. The study was performed in compliance with the Helsinki convention for the use and care of animals. In this study, we used 4 healthy male pigs, aged 36 months, which were fasted for $72 \mathrm{~h}$ at the start of the investigation.

\section{Procedure}

A xipho-pubic laparotomy was performed after sedation and analgesia of the animal by an expert veterinarian. Once the laparotomy was performed, a standard grade $\mathrm{V}$ liver injury, cutting the liver $2.5 \mathrm{~cm}$ in depth, was created with a stellate shape device as described by Holcomb et al. [13] (Figs. 1 and 2 and Additional file 2: Video 2). After that, 2 pigs were treated with PHP (Fig. 3), while the 2 remaining were treated with VBP (Additional file 3: Video 3). 


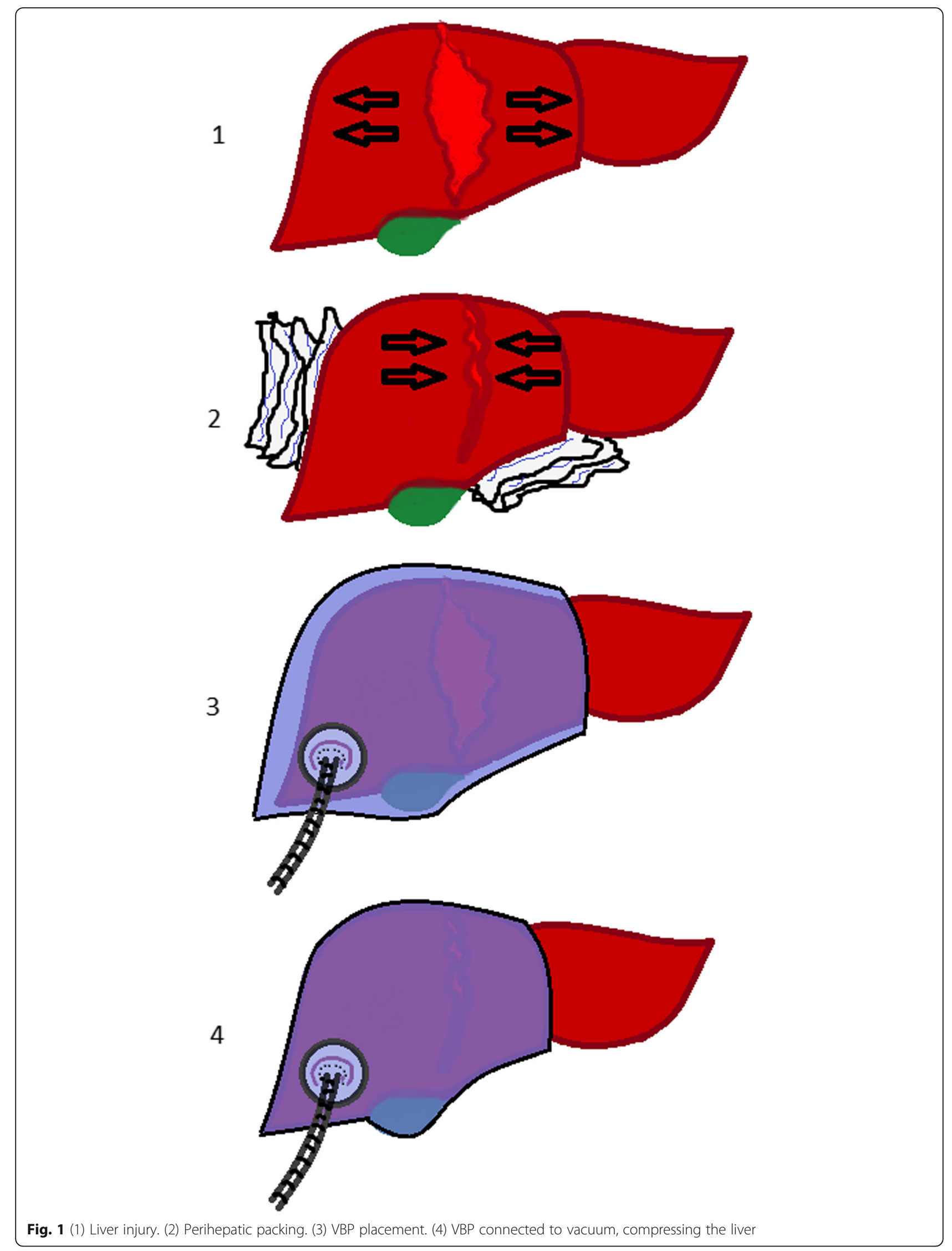




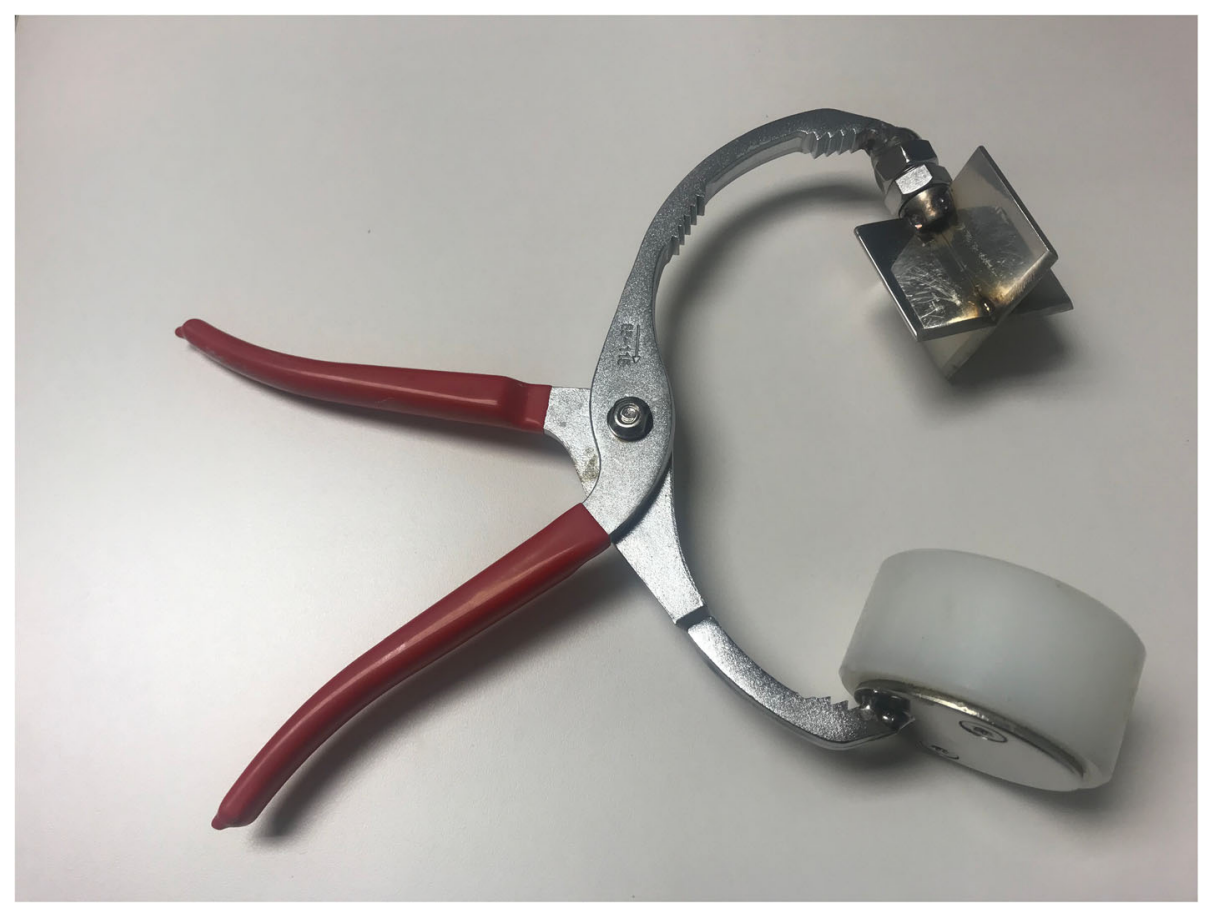

Fig. 2 Stellate shape device used to inflict the liver injury

In the PHP group, compresses were placed around the liver and the abdominal wall was subsequently closured. In the VBP group, the device was placed covering the liver. There is no need to mobilize the liver in the swine model, as the bag can easily cover a full hepatic lobe (Fig. 4). On the contrary, in humans, the liver should be partly mobilized so the bag can completely cover the injury. Once it was in place, the vacuum was established at $75 \mathrm{mmHg}$. After any of the two techniques, the laparotomy was closed using PDS loop 0.

During the surgery, aspiration and quantification of the hemorrhage was performed. In the same way, the hemodynamic stability was determined by means of an anesthesia chart as well as the need or not for vasoactive support.

\section{Variables}

Analytical and coagulation values were determined prior to surgery as well as the reference intra-abdominal pressure (IAP). New IAP was taken after abdominal closure and daily analytical values (blood count, CRP, liver enzymes, coagulation times, fibrinogen, and lactic acid) were recorded.

After $72 \mathrm{~h}$, a reintervention was performed. Both PHP and VBP were retired, and effective hemostasis and free blood in the cavity were assessed. Possible complications of the technique were described. Subsequently, the animals were euthanized in compliance with all the standards.
Continuous variables were reported as median (range), whereas categorical variables were reported as number of patients and percentage. Data were analyzed using Excel $^{\circ}$ (Microsoft 2010).

\section{Results \\ Feasibility}

The device was correctly placed without any issues during the active bleeding. Both VBP cases maintained negative pressure during $72 \mathrm{~h}$ with no leaks. After $72 \mathrm{~h}$, the device was correctly recovered. There was no incidence of bleeding or bile leak after removal.

\section{Intraoperative and postoperative parameters}

In the PHP group, one of the pigs did not survive the first $24 \mathrm{~h}$. Both 2 pigs in the VBP group and the remaining one in the PHP group went through the full 72-h period and were successfully reoperated.

Intraoperative and postoperative parameters are exposed in Table 1. As can be seen, HR was higher in the PHP group (103 bpm) than in the VBP group (82 bpm). Temperature, IAP, and fluid requirements were similar.

\section{Blood tests}

Blood test results are exposed in Table 2. Both $\mathrm{Hb}$ and hematocrit results were higher in the VBP group, while leukocytosis was lower. There were no substantial differences in CPR, coagulation, and liver enzymes. 


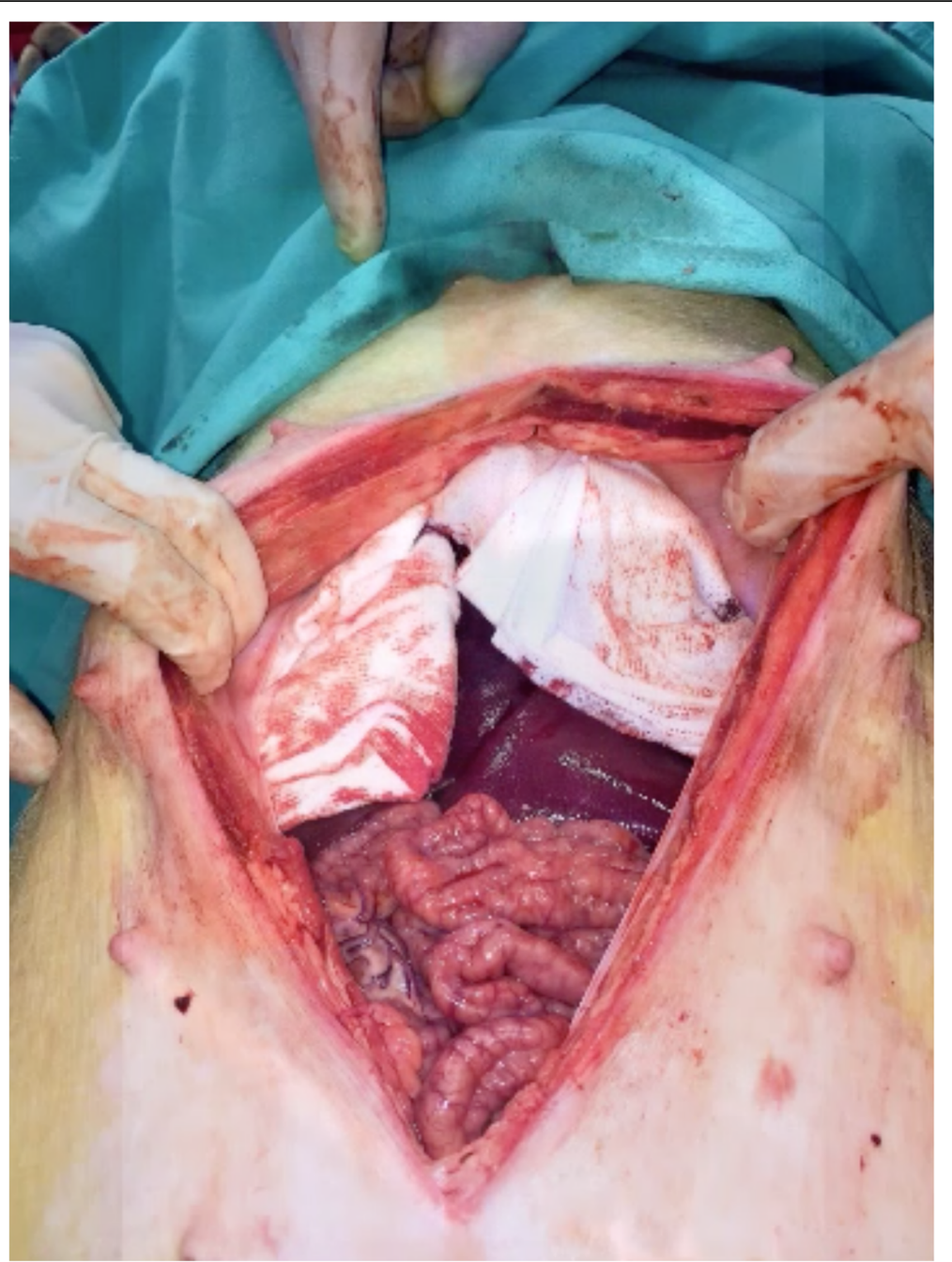

Fig. 3 Perihepatic packing in the swine model

\section{Discussion}

The liver is the most injured organ following abdominal trauma and the first cause of death after suffering an abdominal injury [14].

Poor outcomes of hepatic resections in the emergency setting and the understanding of the hemorrhagic shock have led us towards the concept of damage control laparotomy with perihepatic packing. This approach, combined with advances in resuscitation and interventional radiology, has delivered a decline in overall mortality rates [15].

Perihepatic packing consists of placing compresses under pressure around the liver intended to contain the bleeding and to promote coagulation. The compresses are placed manually during the first surgical intervention. The liver is wrapped with pressure subjectively determined by the surgeon who places the compresses, based on their experience. Approximately $48-72 \mathrm{~h}$ after the placement of the compresses, a liver condition check is performed to check if bleeding persists. The previously placed packing is removed, and depending on the state of the liver, it can be left that way, a new surgical procedure could be needed, or in case of persisting bleeding, a new packing is placed.

Although hepatic packing is an extended maneuver, it requires experience to perform it properly and involves several potential complications. An excessive packing can cause secondary vena cava compression, which can decrease cardiac output and potentially cause death in hypotensive patients after hemorrhagic shock [2, 7]. Excessive pressure can also provoke abdominal compartment syndrome [8] with compromise of splanchnic perfusion, renal failure, heart failure, respiratory failure, and potentially death. However, a poorly placed liver packing with too low pressure may be ineffective, and thus, bleeding can continue inadvertently with the consequent hypovolemic shock and potentially death $[2,7,9]$. 


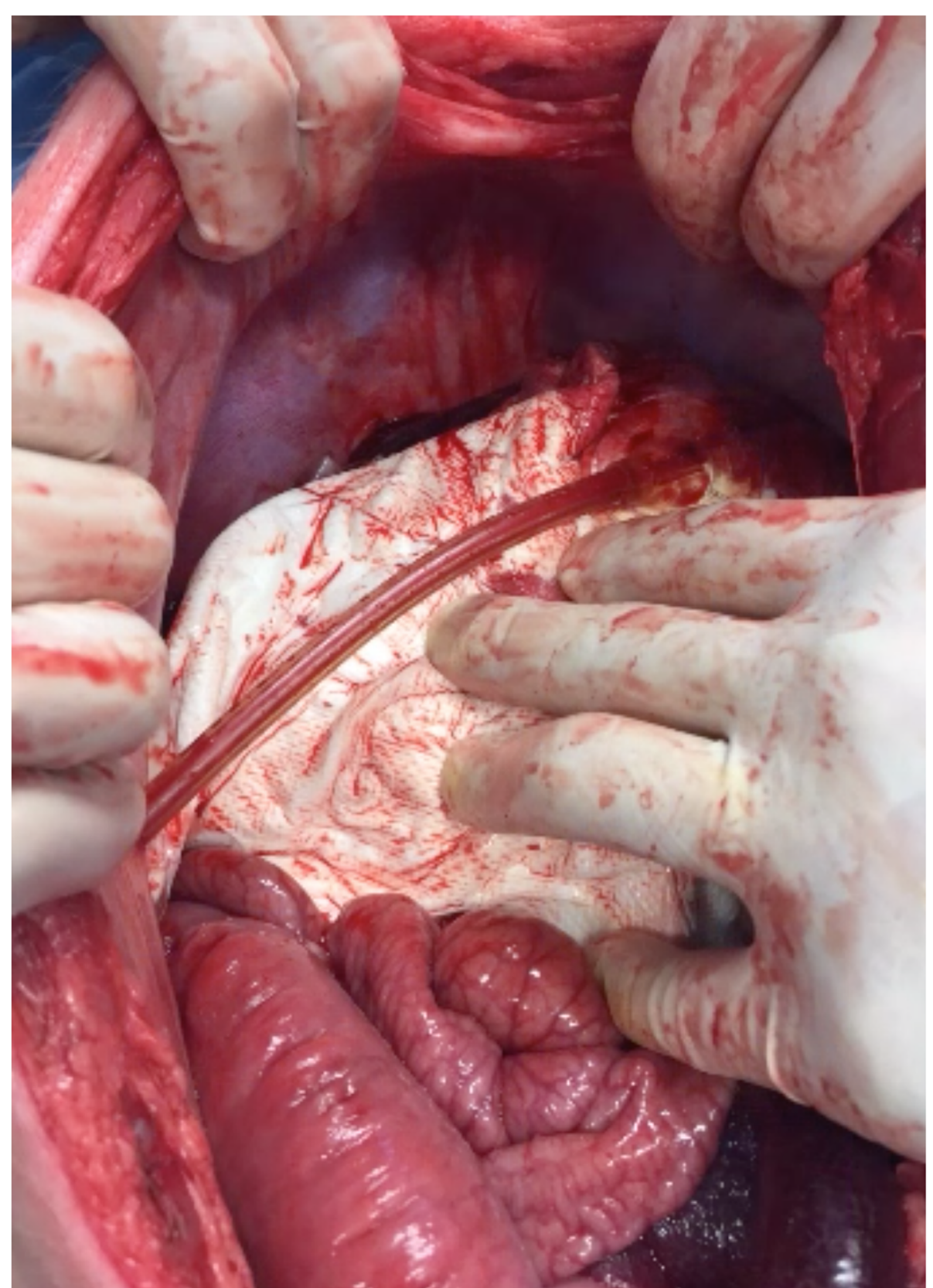

Fig. $4 \mathrm{VBP}$ placed and working in the swine model

Even if bleeding control is achieved through this maneuver, subsequent complications such as intraabdominal abscesses, bleeding after withdrawal, or the loss of compresses within the patient are still common.

In short, there is a need for improvements in this field to allow a simpler and safer liver packing. In particular, there is a need for devices that allow an objective way to control the pressure applied to the liver, to monitor blood debit after abdominal closure, and to prevent the loss of material within the abdominal cavity.
Inserting an organ in the bag is a relatively easy procedure that can be performed by surgeons who are not necessarily experts in abdominal trauma nor in liver surgery. The bag may thus be used as a first treatment for controlling hemorrhages in abdominal traumas. These can take place everywhere, even in places where there are no surgeons with a high expertise in abdominal traumas. Therefore, using this bag may allow for a first and quick treatment, and it provides the opportunity to refer to another center or to perform an angioembolization, 


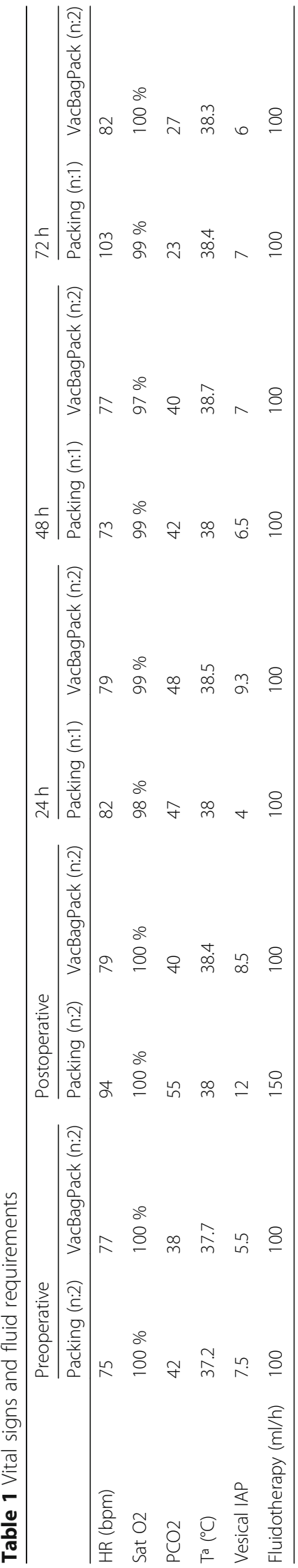




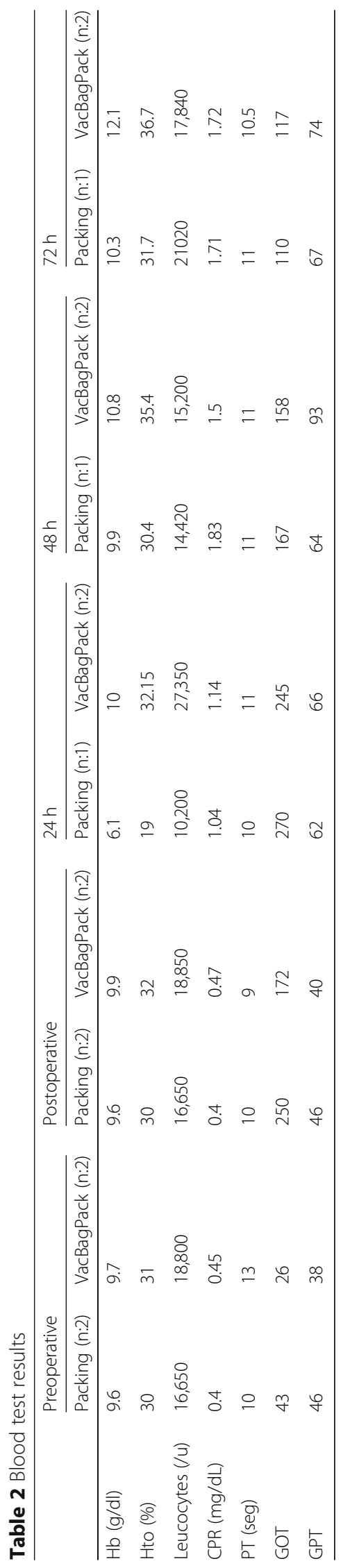


which increases the probability of recovery of the patient.

Our study indicates that placing this new device is feasible. There were no leaks of the device, and no evidence of increased bleeding or bile leak due to the negative pressure was found, both of which were hypothetic risks of the new device.

Both pigs survived with the new device and showed an equivalent outcome to the one that survived in the traditional technique group. Blood tests were similar too. This suggests that VBP could be at least as effective as traditional PHP.

\section{Conclusions}

This is a first approach and proof of concept; therefore, it has several limitations. The limited sample size in this first pilot experience and the obvious differences between pigs and humans prevent us from drawing conclusions. However, we establish a first step towards the development of this new device. A new study with different injuries in different parts of the liver and a bigger sample size still in pigs will be conducted.

Also, an industrial model of the device is currently in production.

\section{Supplementary information}

Supplementary information accompanies this paper at https://doi.org/10. 1186/s13017-019-0277-7.

Additional file 1. Video 1: Confection of a homemade prototype, the future device will not require this step as it will be a ready-to-use system.

Additional file 2. Video 2: Stellate shape device inflicting the liver injury

Additional file 3. Video 3: VBP placed and activated in order to stop the hepatic bleeding

\section{Abbreviations}

CRP: C-reactive protein; IAP: Intrabdominal pressure; PHP: Perihepatic packing; VBP: VacBagPack device

\section{Acknowledgements}

No acknowledgements.

\section{Authors' contributions}

JJS and XGA designed the original device. JJS, ACM, and CPF designed the study. JJS, ACM, CPF, and RMS performed the surgery and collected the data. JJS and ACM elaborated the manuscript which was revised by RMS and XGA. All authors read and approved the final manuscript.

\section{Funding}

Private funding was received and used to buy the swine model and pay for the theater hours. Authors and researchers received no money.

This research has received funding from local authorities (IbSalut), as a research scholarship, and from Swan Medical. Acknowledgment to IDISBA for contributing to its publication in Open Access.

\section{Availability of data and materials}

Excel documents' photographies and videos are available.
Consent for publication

All authors gave their approval on the final version for publication.

\section{Competing interests}

JSS and XGA are authors of the original patent which describes the device. The other authors declare that they have no competing interests.

\section{Author details}

${ }^{1}$ General \& Digestive Surgery Department, University Hospital Son Espases, Palma de Mallorca, Spain. ${ }^{2}$ Health Research Institute of Balearic Islands, Palma de Mallorca, Spain. ${ }^{3}$ School of Medicine, University of Balearic Islands, Palma de Mallorca, Spain.

Received: 17 October 2019 Accepted: 18 November 2019 Received: 17 October 2019 Accepted: $18 \mathrm{Nov}$

References

1. Doklestić K, Stefanović B, Gregorić $P$, Ivančević $N$, Lončar $Z$, Jovanović B, et al. Surgical management of AAST grades III-V hepatic trauma by Damage control surgery with perihepatic packing and Definitive hepatic repairsingle centre experience. World J Emerg Surg. 2015;10:34.

2. Coccolini F, Montori G, Catena F, Di Saverio S, Biffl W, Moore EE, et al. Liver trauma: WSES position paper. World J Emerg Surg. 2015;10:39.

3. Asensio JA, Petrone P, García-Núñez L, Kimbrell B, Kuncir E. Multidisciplinary approach for the management of complex hepatic injuries AAST-OIS grades IV-V: a prospective study. Scand J Surg. 2007;96:214-20.

4. Peitzman $A B$, Richardson JD. Surgical treatment of injuries to the solid abdominal organs: a 50-year perspective from the Journal of Trauma. J Trauma. 2010;69:1011-21.

5. Tinkoff G, Esposito TJ, Reed J, Kilgo P, Fildes J, Pasquale M, et al. American Association for the Surgery of Trauma Organ Injury Scale I: Spleen, Liver, and Kidney, Validation Based on the National Trauma Data Bank. J Am Coll Surg. 2008;207:646-55.

6. Reed RL, Merrell RC, Meyers WC, Fischer RP. Continuing evolution in the approach to severe liver trauma. Ann Surg. 1992;216:524-38.

7. Ward J, Alarcon L, Peitzman AB. Management of blunt liver injury: what is new? Eur J Trauma Emerg Surg. 2015;41:229-37.

8. Gao J, Du D, Zhao X, Liu G, Yang J, Zhao S, et al. Liver trauma: experience in 348 cases. World J Surg. 2003;27:703-8.

9. Aydin U, Yazici P, Zeytunlu M, Coker A. Is it more dangerous to perform inadequate packing? World J Emerg Surg. 2008;3:1.

10. Segura-Sampedro JJ, Gonzalez-Argente X, Cañete-Gómez J, Reguera-Rosal J. Device for organ packing. 2019: WO 2019/137931 A1.

11. Eschbach D, Steinfeldt T, Hildebrand F, Frink M, Schöller K, Sassen M, et al A porcine polytrauma model with two different degrees of hemorrhagic shock: outcome related to trauma within the first $48 \mathrm{~h}$. Eur J Med Res. 2015;20:1-14.

12. Grottke $\mathrm{O}$, Braunschweig T, Philippen B, Gatzweiler KH, Gronloh N, Staat M, et al. A new model for blunt liver injuries in the swine. Eur Surg Res. 2010; 44:65-73.

13. Holcomb JB, Pusateri AE, Harris RA, Charles NC, Gomez RR, Cole JP, et al. Effect of dry fibrin sealant dressings versus gauze packing on blood loss in grade $V$ liver injuries in resuscitated swine. J Trauma. 1999;46:49-57.

14. Badger SA, Barclay R, Campbell P, Mole DJ, Diamond T. Management of liver trauma. World J Surg. 2009;33:2522-37.

15. Tarchouli M, Elabsi M, Njoumi N, Essarghini M, Echarrab M, Chkoff MR. Liver trauma: what current management? Hepatobiliary Pancreat Dis Int. 2018;17: $39-44$.

\section{Publisher's Note}

Springer Nature remains neutral with regard to jurisdictional claims in published maps and institutional affiliations. 